\title{
Current Pharma News
}

Bowel Cancer: Low-calorie soft drinks could reduce recurrence

Published: July 20, 2018

By Catharine Paddock PhD

Consuming artificially sweetened soft drinks such as diet colas may be linked to a significantly lower risk of cancer return or death in patients with stage 3 colorectal cancers.

\section{Are essential oils safe for babies?}

Last reviewed Friday 20 July 2018

By Amanda Barrell

Reviewed by Carissa Stephens, RN, CCRN, CPN

There is some evidence for the benefits of essential oils, but very little research on how these oils may affect babies. It is crucial to note that the American Association of Naturopathic Physicians do not recommend using essential oils at all on babies younger than 3 months.

How long does nicotine stay in your system?

Last reviewed Thursday 19 July 2018

By Danielle Dresden

Reviewed by Alan Carter, PharmD

When people use tobacco products, some of the nicotine stays in their system after they quit smoking. Medical tests can detect nicotine in people's urine, blood, saliva, hair and nails. Nicotine is the addictive substance in tobacco, cigarettes and vapes or ecigarettes.

When someone smokes a cigarette, their body absorbs up to 90 percent of the nicotine. Traces of nicotine will linger long after individuals no longer feel the effects. After smoking a cigarette, nicotine and its by-products stay in a person's urine and saliva for 4 days and blood for up to 10 days.

\section{Eating dinner earlier could reduce cancer risk}

Published Thursday 19 July 2018

By Tim Newman

According to a study that was conducted at the Barcelona Institute for Global Health in Spain, eating your final meal of the day too late can increase the risk of developing cancer.

The relationship between food and cancer has been investigated to a great deal. For instance, regularly eating fresh vegetables has been shown to reduce cancer risk. Conversely, regularly eating red meat increases the risk of certain cancers.

A recent study investigated potential links between meal timing and two common types of cancer: prostate cancer and breast cancer.

\section{Source: medicalnewstoday.com}

Non-dairy beverages like soy and almond milk may not be 'milk,' FDA suggests

By Lindsey Ellefson, CNN

Updated: July 19, 2018

$(C N N)$ Got milk? If you're buying "milk" made with non-dairy products like almonds or oats, the US Food and Drug Administration isn't so sure you do.

During a the Politico Pro Summit on Tuesday, FDA Commissioner Dr. Scott Gottlieb questioned whether the "standards of identity" applied to milk in the United States are being enforced correctly.

The FDA describes milk as "the lacteal secretion, practically free from colostrum, obtained by the complete milking of one or more healthy cows." That definition doesn't leave room for vegan alternatives to call themselves "milk," though a number of products on the market do.

Non-dairy milks, including soy, almond, rice and coconut milk are juices from nuts, seeds, grains and legumes that may be fortified with vitamins and minerals to deliver the equivalent nutrient profile and sometimes taste and consistency of cow's milk.

\section{Source: cnn.com/health}

The risk of having a heart attack while pregnant, giving birth, or during the two months after delivery, continues to increase for American women, a new study finds. 
As published online July 18 in the Mayo Clinic Proceedings, the study, led by NYU School of Medicine researchers, found that the risk of suffering a heart attack among pregnant women rose 25 percent from 2002 to 2014.

The researchers suggest that the trend among many women to have children later in life is one possible reason for the increase, as heart attack risk rises with age overall, and especially during pregnancy. More women, they say, are also obese and/or have diabetes, key risk factors for heart attack. Another factor that may explain the rising numbers is that myocardial infarcts, the technical name for heart attacks, are easier to detect than a decade ago, as tests for early protein markers of related heart cell damage have improved and become more widely available.

Source: sciencedaily.com

Information Collected and complied by:

Md. Akbar Hossain

Department of Pharmacy

ASA University Bangladesh 